\title{
Multiplicity and forward energy fluctuations from the NA61/SHINE experiment at CERN
}

\section{Andrey Seryakov ${ }^{1}$ for the NA61/SHINE collaboration}

St.Petersburg State University

13B Universitetskaya Emb., St Petersburg 199034, Russia

E-mail: seryakoveyahoo.com

Preliminary results for multiplicity and forward energy fluctuations are presented for inelastic $p+p$ and the most central ${ }^{7} \mathrm{Be}+{ }^{9} \mathrm{Be}$ and ${ }^{40} \mathrm{Ar}+{ }^{45} \mathrm{Sc}$ collisions. The data were obtained by the NA61/SHINE detector at the CERN SPS. Centrality selection and forward energy measurement are based on the nucleon spectator energy in the forward hemisphere determined by the projectile spectator detector. The scaled variance $\omega$ of the multiplicity distribution and the strongly intensive quantity $\Omega$ of multiplicity and forward energy fluctuations were calculated for negatively charged hadrons. The scaled variance changes little when going from $\mathrm{p}+\mathrm{p}$ to $\mathrm{Be}+\mathrm{Be}$ collisions and drops dramatically from $\mathrm{Be}+\mathrm{Be}$ to $\mathrm{Ar}+\mathrm{Sc}$ interactions. A comparison with the EPOS event generator is shown.

Critical Point and Onset of Deconfinement - CPOD2017

7-11 August, 2017

Location

The Wang Center, Stony Brook University, Stony Brook, NY

\section{${ }^{1}$ Speaker}




\section{Introduction}

This paper presents preliminary results referring to event-by-event multiplicity fluctuations of negatively charged hadrons produced in inelastic $\mathrm{p}+\mathrm{p}$ and the most central $\mathrm{Ar}+\mathrm{Sc}$ and $\mathrm{Be}+\mathrm{Be}$ collisions from the NA61/SHINE collaboration. The strong interactions programme of NA61/SHINE, a fixed-target experiment at the CERN SPS, is dedicated to the study of the properties of deconfinement and the search for a critical point of strongly interacting matter. The study of different fluctuation measures is supposed to be a good approach in this search. In particular, multiplicity fluctuations are assumed to reveal a non-monotonic behaviour in the vicinity of the critical point [1].

The paper is organized as follows. In sec.2 there is an introduction of two measures of multiplicity fluctuations: the scaled variance $\omega$ and the strongly intensive quantity $\Omega$ [2]. The event and centrality selection procedure is discussed in sec.3. Sec. 4 and sec.5 contains the description of the NA61/SHINE acceptance, track selection, uncertainties and corrections. Results are presented and discussed in sec. 6 and sec. 7 respectively.

\section{Multiplicity and forward energy fluctuations}

To study multiplicity fluctuations one often uses the scaled variance $\omega$, an intensive quantity, of the multiplicity distribution:

$$
\omega[\mathrm{N}]=\frac{\left\langle\mathrm{N}^{2}\right\rangle-\langle\mathrm{N}\rangle^{2}}{\langle\mathrm{~N}\rangle}
$$

where $\mathrm{N}$ is the multiplicity of charged particles in an event, and \langle\rangle denotes the averaging over events. As a ratio of extensive event quantities, $\omega$ neither depends on the volume of the system within the grand canonical ensemble (GCE) of statistical mechanics nor on the number of sources within models of independent sources like the wounded nucleon model (WNM). The scaled variance has the value zero if there are no multiplicity fluctuations and unity for a Poisson multiplicity distribution.

As the scaled variance is still sensitive to volume fluctuations [3] and the volume of the system created in heavy ion collisions cannot be explicitly fixed in the experiment, one should find a way to reduce the contribution from these trivial fluctuations. The analysis presented below follows two different approaches to minimize this effect:

- selection of narrow centrality classes to constrain fluctuations of the number of nucleonparticipants (see next section).

- study of fluctuations in terms of strongly intensive quantities, which do not depend on eventby-event volume fluctuations (in GCE and WNM) by definition [2-3].

The current analysis uses the strongly intensive quantity $\Omega[\mathrm{A}, \mathrm{B}]$ :

$$
\Omega[\mathrm{A}, \mathrm{B}]=\omega[\mathrm{A}]-\frac{\langle\mathrm{AB}\rangle-\langle\mathrm{A}\rangle\langle\mathrm{B}\rangle}{\langle\mathrm{B}\rangle}
$$

It was shown in Ref. [2] that if A and B are uncorrelated from the fixed volume of the GCE or a single source in the WNM then $\Omega[\mathrm{A}, \mathrm{B}]$ is equal to the scaled variance of the quantity $\mathrm{A}$ from the fixed volume (GCE) or a single source (WNM). To obey this condition A is selected as the multiplicity of produced particles and $B$ as the projectile participant energy $E_{p}=E_{b e a m}-E_{P S D}$, where $E_{\text {beam }}$ is the total beam energy and $E_{\mathrm{PSD}}$ is the energy measured by the PSD, the NA61/SHINE hadron calorimeter positioned exactly on the beam line [4]. 


\section{Event selection and centrality determination}

The centrality selection procedure is a crucial part of any fluctuation study. As a fixed-target experiment, NA61/SHINE has the unique possibility to measure the total energy of noninteracting projectile nucleons. In order to perform this, the hadron calorimeter PSD was placed right on the beam line downstream of the TPCs [4]. Consisting of 44 independent modules in the transverse plane, the PSD detector provides the possibility to minimize the contribution of produced particles to the calorimeter signal and maximize that of projectile nucleon spectators by selecting different sets of modules for each colliding system and energy.

In order to reduce autocorrelation between multiplicity of charged particles used for fluctuation analysis and signals utilized for the centrality determination, only the energy deposited in the PSD calorimeter is used for event selection in the NA61/SHINE experiment. Event-byevent multiplicity measured by the TPCs does not take part in the procedure.

The exact way of experimental centrality determination has a large effect on the multiplicity fluctuation measurement. Therefore, for a proper comparison between models and data, theoreticians have to repeat the experimental procedures as closely as possible, because quantities obtained with centrality selection based on multiplicity, impact parameter or forward energy may differ a lot, especially for light and intermediate size nuclei such as Be and Ar. The NA61/SHINE centrality selection procedure is fully based on forward energy. To calculate this energy in a Monte-Carlo event generator, the PSD acceptance maps [5] should be used. Then the 5\% of the events with the lowest energy in the acceptance will correspond to the NA61/SHINE results for $5 \%$ centrality selection.

\section{Track selection and acceptance}

Track selection criteria were chosen to select only primary charged hadrons and reduce the contamination from secondary interactions and weak decays. Moreover, tracks were restricted to transverse momentum $\mathrm{p}_{\mathrm{T}}<1.5 \mathrm{GeV} / \mathrm{c}$ and rapidity (calculated with pion mass assumption) in the range $0<y_{\pi}<y_{\text {beam }}$.

Since multiplicity fluctuations strongly depend on the acceptance in which the quantities of interest are measured, the same acceptance should be used for any model comparison. The NA61/SHINE acceptance maps can be found in Ref. [6].

\section{Statistical and systematic uncertainties, corrections}

Statistical uncertainties were calculated using the sub-sample method. Analysis of systematic uncertainties is in progress and their values are expected to be smaller than $5 \%$. Corrections for experimental biases have not yet been applied for the presented $\mathrm{Ar}+\mathrm{Sc}$ and $\mathrm{Be}+\mathrm{Be}$ results. To minimize such biases only results for negatively charged hadrons are shown. Nevertheless, to estimate the magnitudes of detector biases, an analysis of events generated by the EPOS event generator was performed at two levels: before and after detector response simulated with the GEANT based NA61/SHINE program chain. A comparison of the results is shown in Fig. 1. The differences between results are much smaller than 5\% except at the top energy where they are on the level of $5 \%$.

The $p+p$ data is fully corrected for detector inefficiency and off-target interactions. The detailed description of the procedure can be found in Ref. [7]. 

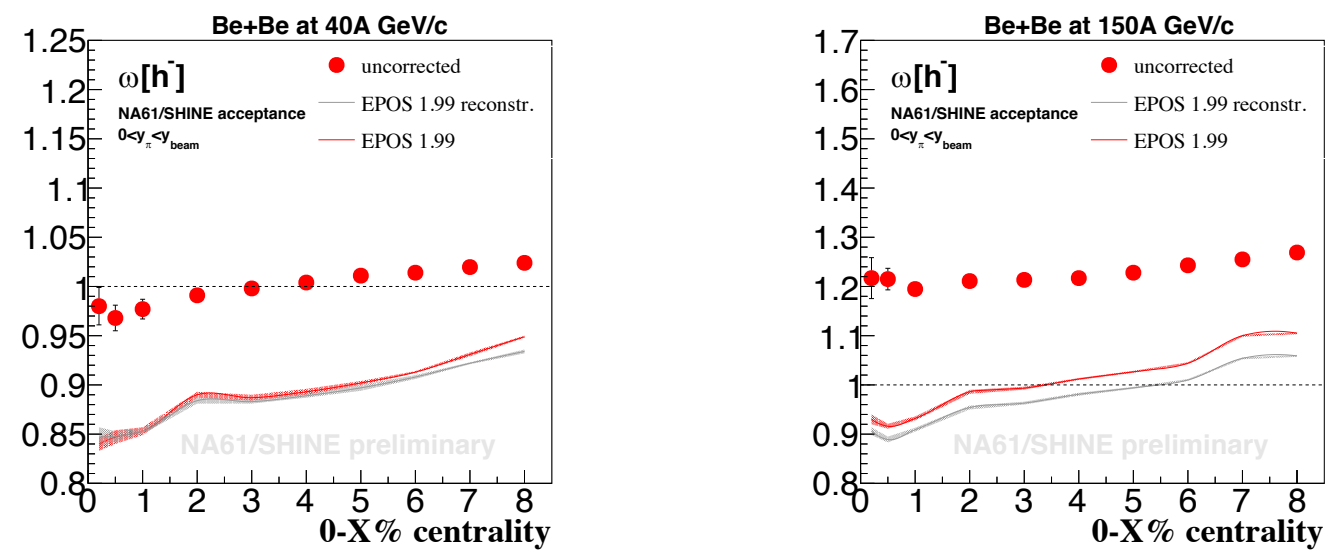

Figure 1. Centrality dependence of $\omega\left[\mathrm{h}^{-}\right]$in Be+Be collisions at $40 A$ (left) and $150 A$ (right) GeV/c. Uncorrected preliminary NA61/SHINE data are shown by red dots, pure EPOS predictions in the NA61/SHINE acceptance by the red lines and reconstructed EPOS after detector simulation by the grey lines. The two presented energies show the largest differences between pure and reconstructed simulation.

\section{Results}

Preliminary results, presented here, refer to accepted primary negatively charged hadrons produced in centrality selected $\mathrm{Be}+\mathrm{Be}$ and $\mathrm{Ar}+\mathrm{Sc}$ collisions and in inelastic $\mathrm{p}+\mathrm{p}$ interactions in the acceptance of the NA61/SHINE experiment.

Figure 2 shows that the strongly intensive quantity $\Omega\left[\mathrm{h}^{-}, \mathrm{E}_{\mathrm{P}}\right]$ does not depend on the width of the centrality interval, as expected, in contrast to the scaled variance of the multiplicity distribution. Both quantities approach the same limit for the most central collisions.
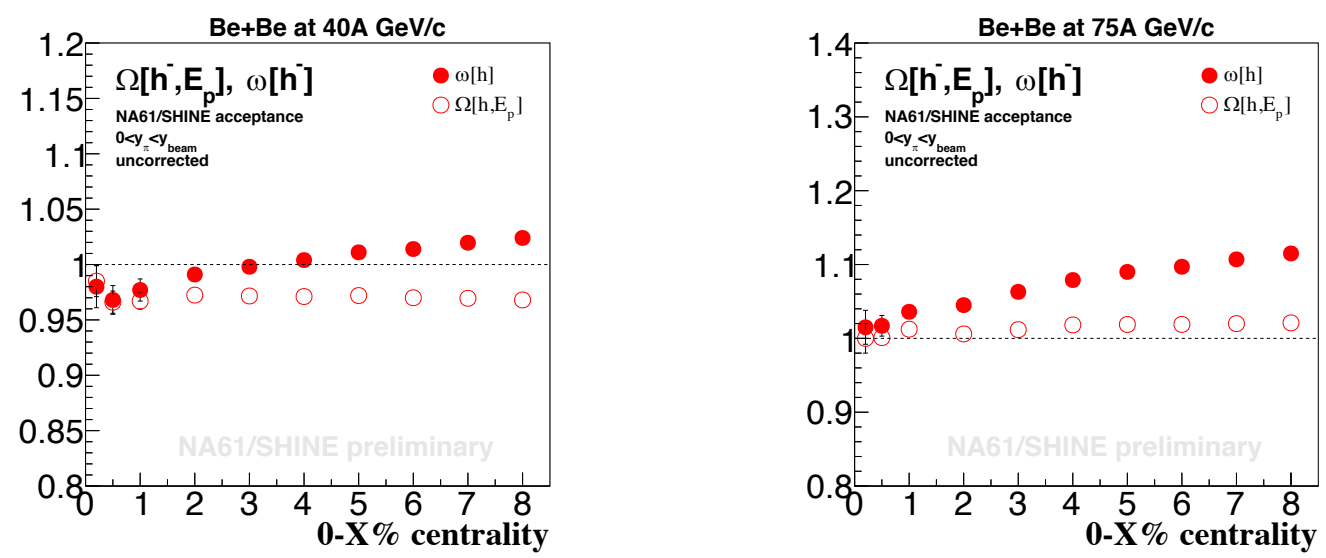

Figure 2. Centrality dependence of $\omega\left[\mathrm{h}^{-}\right]$and $\Omega\left[\mathrm{h}^{-}, \mathrm{E}_{\mathrm{P}}\right]$ in $\mathrm{Be}+\mathrm{Be}$ collisions at $40 A$ (left) and $75 A$ (right) $\mathrm{GeV} / \mathrm{c}$.

The energy dependence of $\omega\left[\mathrm{h}^{-}\right]$and $\Omega\left[\mathrm{h}^{-}, \mathrm{E}_{\mathrm{P}}\right]$ for central Be+Be and Ar+Sc collisions as well as results for $\omega\left[\mathrm{h}^{-}\right]$in $\mathrm{p}+\mathrm{p}$ interactions are displayed by Fig.3. One may notice that the systems created in $\mathrm{p}+\mathrm{p}$ and the most central $\mathrm{Be}+\mathrm{Be}$ collisions show the same rising tendency for all SPS energies. However, the energy dependence for $\mathrm{Ar}+\mathrm{Sc}$ collisions is strikingly different. Fluctuations do not increase with beam energy and are significantly below the $\mathrm{p}+\mathrm{p}$ and $\mathrm{Be}+\mathrm{Be}$ data. 
Figure 4 shows the system size dependence of $\omega\left[\mathrm{h}^{-}\right]$at the highest SPS energy. The results for the light $\mathrm{Be}+\mathrm{Be}$ system is close to that for $\mathrm{p}+\mathrm{p}$ interactions. However, multiplicity fluctuations are significantly suppressed in central $\mathrm{Ar}+\mathrm{Sc}$ collisions. A similar suppression in central $\mathrm{Pb}+\mathrm{Pb}$ collisions was previously observed in Ref. [7]. The EPOS model reproduces the suppression in the $\mathrm{Ar}+\mathrm{Sc}$ system, but also expects it in $\mathrm{Be}+\mathrm{Be}$ collisions.

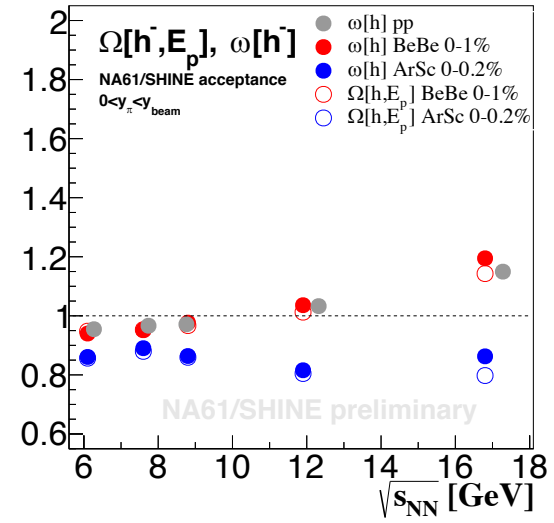

Figure 3. Energy dependence of negatively charged hadron multiplicity fluctuations obtained by NA61/SHINE at forward rapidity $0<\mathrm{y}_{\pi}<\mathrm{y}_{\text {beam }}$ and transverse momenta $\mathrm{p}_{\mathrm{T}}<1.5 \mathrm{GeV} / \mathrm{c}$. The scaled variance is shown for inelastic $\mathrm{p}+\mathrm{p}$ (grey dots), $0-1 \% \mathrm{Be}+\mathrm{Be}$ (full red dots) and $0-0.2 \%$ $\mathrm{Ar}+\mathrm{Sc}$ (full blue dots) collisions and the strongly intensive quantity $\Omega\left[\mathrm{h}^{-}, \mathrm{E}_{\mathrm{P}}\right]$ by corresponding open dots. Only statistical uncertainties are shown.

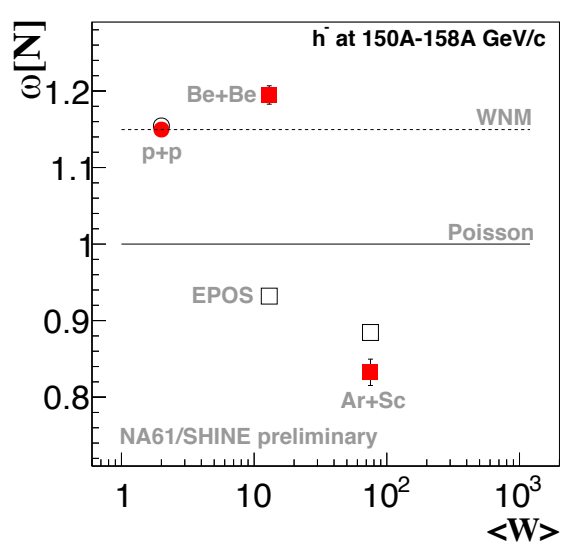

Figure 4. Scaled variance of the multiplicity distribution of negatively charged hadrons versus mean number of wounded nucleons at high SPS energy. Results for inelastic $\mathrm{p}+\mathrm{p}$ interactions as well as central $0-1 \% \mathrm{Be}+\mathrm{Be}$ and $0-0.2 \% \mathrm{Ar}+\mathrm{Sc}$ collisions are shown at forward-rapidity, $0<\mathrm{y}_{\pi}<\mathrm{y}_{\text {beam }}$, and in $\mathrm{p}_{\mathrm{T}}<1.5 \mathrm{GeV} / \mathrm{c}$. Only statistical uncertainties are shown. The NA61/SHINE data are compared to the EPOS1.99 model predictions (open squares).

\section{Conclusions}

New results of multiplicity fluctuation measurements performed by the NA61/SHINE experiment at CERN SPS energies were presented in terms of the intensive quantity $\omega$ and the strongly intensive quantity $\Omega$.

In the most central collisions the values of these two measures approaches the same limit as expected. The value of $\omega$ increases with the width of the centrality interval in contrast to the constant value of $\Omega$. Thus, the strongly intensive quantity $\Omega$ better characterizes features of individual sources in heavy ion collisions.

Unexpectedly, the light nuclei system $(\mathrm{Be}+\mathrm{Be})$ shows fluctuations of multiplicity very similar to those in $\mathrm{p}+\mathrm{p}$ interactions, while they are significantly suppressed in central $\mathrm{Ar}+\mathrm{Sc}$ collisions. A suppression of multiplicity fluctuations in the most central $\mathrm{Pb}+\mathrm{Pb}$ collisions compared to $p+p$ reactions was previously observed in Ref. [7]. Therefore, the new results from $\mathrm{Ar}+\mathrm{Sc}$ collisions confirm the effect even for intermediate mass nuclei.

If the suppression is due to different properties of particle sources in $p+p$ and $\mathrm{Be}+\mathrm{Be}$ collisions versus $\mathrm{Ar}+\mathrm{Sc}$ reactions, then this can be considered as an indication of some type of transition in heavy ion collisions, namely, a rapid change of hadron production properties when moving from the $\mathrm{Be}+\mathrm{Be}$ to the $\mathrm{Ar}+\mathrm{Sc}$ colliding system. The effect can be interpreted as the beginning of creation of large clusters of strongly interacting matter and may be explained by 
percolation models [8-12] or AdS/CFT correspondence [13]. This experimental phenomenon was referred to as the onset of fireball (by Edward Shuryak at the CPOD 2017 conference).

\section{Acknowledgements}

This work was supported by the Russian Science Foundation under grant 17-72-20045.

\section{References}

[1] M. Gazdzicki and P. Seyboth, APhysPolB. 47 (2016) 1201, arXiv:1506.08141[nucl-ex]

[2] R. V. Poberezhnyuk, M. I. Gorenstein, M. Gazdzicki, APhysPolB. 47 (2016) 2055, arXiv:1509.06577v2 [hep-ph]

[3] M. I. Gorenstein and M. Gazdzicki, Phys. Rev. C84 (2011) 014904, 1101.4865

[4] N. Abgrall et al., [NA61/SHINE Collab.] JINST 9 (2014) P06005, arXiv:1401.4699 [physics.insdet]

[5] PSD acceptance maps used for forward energy calculations: https://edms.cern.ch/document/1867336/1

[6] NA61/SHINE acceptance maps used for multiplicity calculations: https://edms.cern.ch/document/1549298/1.

[7] A. Aduszkiewicz et al., [NA61/SHINE Collab.] Eur.Phys.J. C76 (2016) no.11, 635, arXiv: 1510.00163 [hep-ex]

[8] G. Baym, Physica A 96 (1979) 131-135

[9] T. Celik, F. Karsch, and H. Satz, Phys. Lett. 97B (1980) 128-130

[10] M. Braun and C. Pajares, Nucl. Phys. B390 (1993) 542-558

[11] N. Armesto, M. A. Braun, E. G. Ferreiro, and C. Pajares, Phys. Rev. Lett. 77 (1996) 3736-3738, arXiv:hep-ph/9607239 [hep-ph]

[12] L. Cunqueiro, E. G. Ferreiro, F. del Moral, and C. Pajares, Phys. Rev. C72 (2005) 024907, arXiv:hep-ph/0505197 [hep-ph]

[13] S. Lin and E. Shuryak, Phys. Rev. D79 (2009) 124015, arXiv:0902.1508 [hep-th] 